\title{
Dephasing time of GaAs electron-spin qubits coupled to a nuclear bath exceeding $200 \mu \mathrm{s}$
}

\author{
Hendrik Bluhm ${ }^{1 \dagger}$, Sandra Foletti ${ }^{1 \dagger}$, Izhar Neder ${ }^{1}$, Mark Rudner ${ }^{1}$, Diana Mahalu ${ }^{2}$, Vladimir Umansky ${ }^{2}$ \\ and Amir Yacoby ${ }^{1 \star}$
}

Qubits, the quantum mechanical bits required for quantum computing, must retain their quantum states for times long enough to allow the information contained in them to be processed. In many types of electron-spin qubits, the primary source of information loss is decoherence due to the interaction with nuclear spins of the host lattice. For electrons in gate-defined GaAs quantum dots, spin-echo measurements have revealed coherence times of about 1 us at magnetic fields below $100 \mathrm{mT}$ (refs 1,2). Here, we show that coherence in such devices can survive much longer, and provide a detailed understanding of the measured nuclearspin-induced decoherence. At fields above a few hundred millitesla, the coherence time measured using a singlepulse spin echo is $30 \mu \mathrm{s}$. At lower fields, the echo first collapses, but then revives at times determined by the relative Larmor precession of different nuclear species. This behaviour was recently predicted ${ }^{3,4}$, and can, as we show, be quantitatively accounted for by a semiclassical model for the dynamics of electron and nuclear spins. Using a multiple-pulse Carr-Purcell-Meiboom-Gill echo sequence, the decoherence time can be extended to more than $200 \mu \mathrm{s}$, an improvement by two orders of magnitude compared with previous measurements ${ }^{1,2,5}$.

The promise of quantum-dot spin qubits as a solid-state approach to quantum computing is demonstrated by the successful realization of initialization, control and single-shot readout of electron-spin qubits in GaAs quantum dots using optical ${ }^{6}$, magnetic $^{7}$ and fully electrical ${ }^{8-10}$ techniques. To further advance spin-based quantum computing, it is vital to mitigate decoherence due to the interaction of the electron spin with the spins of nuclei of the host material. Understanding the dynamics of this system is also of great fundamental interest ${ }^{11,12}$.

Through the hyperfine interaction, an electron spin in a GaAs quantum dot is subjected to an effective magnetic field produced by the nuclear spins. Under typical experimental conditions, this so-called 'Overhauser field' has a random magnitude and direction. Typically, measurements of the coherent electron-spin precession involve averaging over many experimental runs, and thus over many Overhauser field configurations. As a result, the coherence signal is suppressed for evolution times $\tau \gtrsim T_{2}{ }^{*} \approx 10 \mathrm{~ns}$ (refs 1 , 2 ). However, the nuclear spins evolve much more slowly than the electron spins, so that the Overhauser field is nearly static over sufficiently short time intervals. Therefore, one can partially eliminate the effect of the random nuclear field by flipping the electron spin halfway through an interval of free precession, a procedure known as Hahn echo. The random contributions of the Overhauser field to the electron-spin precession before and after the spin reversal then approximately cancel out. For longer evolution times, the effective field acting on the electron spin generally changes over the precession interval. This change leads to an eventual loss of coherence on a timescale determined by the details of the nuclear spin dynamics.

Previous Hahn-echo experiments on lateral GaAs quantum dots have demonstrated spin-dephasing times of around $1 \mu$ s at relatively low magnetic fields up to $100 \mathrm{mT}$ for microwave-controlled ${ }^{2}$ singleelectron spins and electrically controlled ${ }^{1}$ two-electron-spin qubits. For optically controlled self-assembled quantum dots, coherence times of $3 \mu$ s at $6 \mathrm{~T}$ were found ${ }^{5}$. Recent theoretical studies of decoherence due to the hyperfine interaction ${ }^{3,4,13}$ are generally consistent with these experimental results, but predict revivals of the echo signal after several microseconds, similar to those seen in nitrogen-vacancy centres in diamond ${ }^{14}$. This prediction already indicates that the initial decay of the echo does not reflect irreversible decoherence, but is a consequence of the coherent Larmor precession of the nuclei. Theoretical work also predicted much longer coherence times at higher external magnetic fields ${ }^{13,15}$ or when using more advanced pulse sequences ${ }^{16,17}$. The classic example is the Carr-Purcell-Meiboom-Gill (CPMG) sequence ${ }^{1,18}$, but several alternatives have recently been developed ${ }^{19,20}$ and demonstrated $^{21,22}$. Their performance is expected to improve as more control pulses are added ${ }^{17}$. Here, we provide direct experimental confirmations for all of the above predictions.

The spin qubit studied in this work consists of two isolated electrons confined in a double quantum dot, created by applying negative voltages to metallic gates that locally deplete a twodimensional electron gas $90 \mathrm{~nm}$ below the wafer surface (see Fig. 1a). The Hilbert space of our logical qubit is spanned by the states $|\uparrow \downarrow\rangle$ and $|\downarrow \uparrow\rangle$, that is, the $m=0$ subspace of two separated spins. The arrows represent the alignment of the electron spins in each of the dots relative to an external magnetic field, $B_{\text {ext }}$, which lies in the plane of the two-dimensional electron gas and defines the $z$ direction. The remaining two states, $T_{+} \equiv|\uparrow \uparrow\rangle$ and $T_{-} \equiv|\downarrow \downarrow\rangle$, are energetically separated by the Zeeman energy $E_{\mathrm{Z}}=g^{*} \mu_{\mathrm{B}} B_{\text {ext }}\left(g^{*}=-0.44\right.$ is the $g$-factor in GaAs $)$ and are not used for information storage. Tunnel coupling to the leads is used for initialization and inter-dot tunnelling allows spin exchange between the dots. This exchange interaction is modulated by means of the detuning $\varepsilon$, which is the difference between the electrostatic potentials in the two dots. This parameter is controlled by means of rapid, antisymmetric changes of the voltages on gates GL and GR (see Fig. 1) applied through high-frequency coaxial lines, which enables fast electrical control of the qubit ${ }^{1,8,23}$.

\footnotetext{
${ }^{1}$ Department of Physics, Harvard University, Cambridge, Massachusetts 02138, USA, ${ }^{2}$ Braun Center for Submicron Research, Department of Condensed Matter Physics, Weizmann Institute of Science, Rehovot 76100, Israel. †These authors contributed equally to this work.

*e-mail: yacoby@physics.harvard.edu.
} 


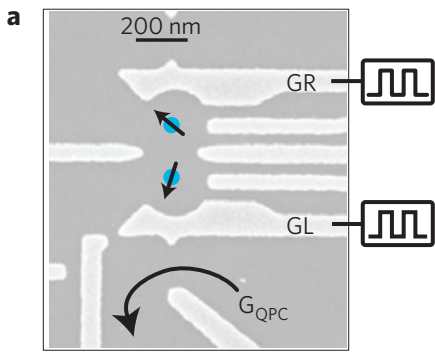

b
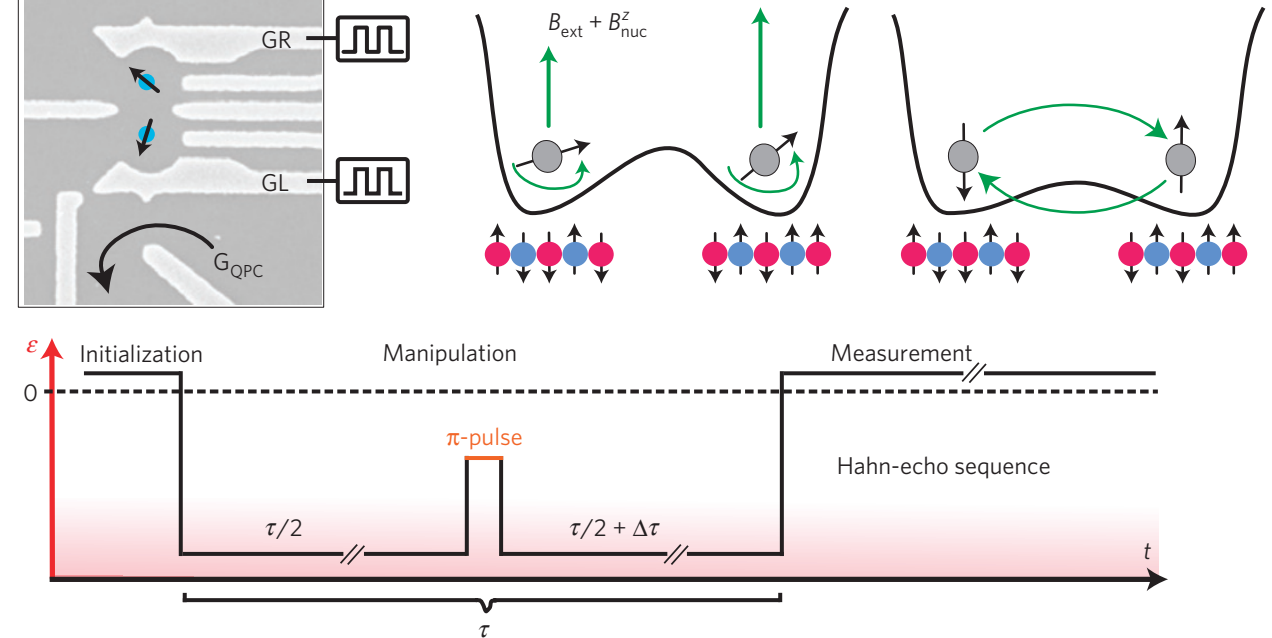

d

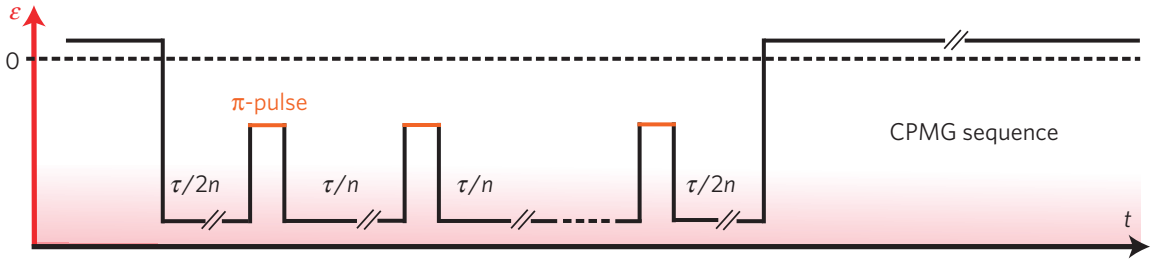

Figure 1 | Qubit control. a, Scanning electron micrograph of a device similar to the one used. Metal gates (bright structures) are negatively biased to confine two electrons. The charge state of the double quantum dot is determined by measuring the conductance through the capacitively coupled QPC, $G_{Q P C}$. The separation between the two electrons is controlled with nanosecond time resolution using the voltages on GR and GL. b, Left: An initially prepared singlet state oscillates between $S$ and $T_{0}$ with frequency $g^{*} \mu_{B} \Delta B_{\text {nuc }}^{z} / \hbar$, which changes over time as a result of slow fluctuations of the hyperfine field gradient $\Delta B_{\text {nuc }}^{z}$. Right: Switching on the tunnel coupling between the two dots leads to the coherent exchange of the electron spins. $\mathbf{c}$, Hahn-echo sequence. After evolving for a time $\tau / 2$, the two electrons are exchanged with a $\pi$-pulse. The singlet state is recovered after further evolution for another $\tau / 2$, independent of $\Delta B_{\text {nuc }}^{z}$. d, CPMG sequence. In this higher-order decoupling sequence, n $\pi$-pulses at time intervals $\tau / n$ are applied.

The experimental procedures follow those of ref. 1. We initialize the system at a large detuning, where the ground state is a spin singlet with both electrons residing in a single dot. As $\varepsilon$ is swept to negative values, the electrons separate into different dots, thus preparing a singlet $S \equiv(|\uparrow \downarrow\rangle-|\downarrow \uparrow\rangle) / \sqrt{2}$. For very large negative detunings, the electron spins in the two dots are decoupled, and each individually experiences a Zeeman field composed of the homogeneous external field and a fluctuating local hyperfine field. A difference $\Delta B_{\text {nuc }}^{z}$ between the $z$-components of the hyperfine fields in the two dots leads to an energy splitting between the basis states $|\uparrow \downarrow\rangle$ and $|\downarrow \uparrow\rangle$. This splitting causes precession between the singlet $S$ and the triplet $T_{0} \equiv(|\uparrow \downarrow\rangle+|\downarrow \uparrow\rangle) / \sqrt{2}$, and its fluctuations lead to dephasing of the qubit. We implement the echo $\pi$-pulses by pulsing to small negative detunings, where inter-dot tunnelling leads to an exchange splitting between $S$ and $T_{0}$. This splitting drives coherent oscillations between the states $|\uparrow \downarrow\rangle$ and $|\downarrow \uparrow\rangle$. The pulse profiles for the Hahn echo and CPMG sequence are shown in Fig. 1c,d.

Readout of the final qubit state is accomplished by switching to positive detuning, $\varepsilon>0$, where the state with both electrons sitting in the same dot is preferred for the spin singlet, but is energetically excluded for a spin triplet because of the Pauli exclusion principle. The two spin states thus acquire different charge densities. To sense this difference, we use a proximal quantum point contact (QPC), the conductance of which depends on the local electrostatic environment ${ }^{24}$. After averaging over many identical pulse cycles, the mean QPC conductance, $G_{\mathrm{QPC}}$, reflects the probability to find the qubit in the singlet state at the end of each cycle. The echo amplitudes presented below are normalized such that they are unity at short times (no decoherence) and eventually drop to zero for a fully randomized state. This normalization eliminates $\tau$-independent contrast losses (see Supplementary Information). Figure 2a shows the Hahn-echo signals for different magnetic fields $B_{\text {ext }}$.

At high fields we find a decay of the Hahn-echo signal approximately as $\exp \left(-(\tau / 30 \mu \mathrm{s})^{4}\right)$. As the magnetic field is reduced, the echo signal develops oscillations with a timescale of microseconds. For even lower fields, the oscillations evolve into full collapses of the signal, with revivals at later times on a ten-microsecond timescale. These revivals were predicted in refs 3,4 on the basis of a quantum-mechanical treatment of the hyperfine interaction between electron and nuclear spins. Below we outline a semiclassical model that can reproduce the lowestorder result of refs 3,4 and accounts for additional effects that are essential for fitting our data. The derivation is provided in the Supplementary Information and will be discussed in more detail elsewhere. This model provides the theoretical echo signal, $C(\tau) \equiv 2 p(S)-1=-\operatorname{Re}\langle\uparrow \downarrow|\rho(\tau)| \downarrow \uparrow\rangle$, where $p(S)$ is the probability of finding the electron in a singlet state and $\rho(\tau)$ is the density matrix of the qubit at the end of the evolution time. We have used this model to produce the quantitatively accurate fits shown in Fig. 2a.

For each electron spin, the Zeeman-energy splitting is proportional to the total magnetic field $B_{\text {tot }}=\sqrt{\left(B_{\text {ext }}+B_{\text {nuc }}^{z}\right)^{2}+\left(B_{\text {nuc }}^{\perp}\right)^{2}} \approx$ $B_{\text {ext }}+B_{\text {nuc }}^{z}+\left(B_{\text {nuc }}^{\perp}\right)^{2} / 2 B_{\text {ext }}$ (Fig. 2b). Time dependence of the parallel and transverse nuclear components, $B_{\text {nuc }}^{z}$ and $\left(B_{\text {nuc }}^{\perp}\right)^{2}$, can lead to dephasing of the electron spin. Assuming statistical independence between $B_{\text {nuc }}^{z}$ and $\left(B_{\text {nuc }}^{\perp}\right)^{2}$, the theoretical echo signal can be written as a product, $C(\tau)=A_{z}(\tau) R_{\perp}(\tau)$, where $A_{z}(\tau)$ and $R_{\perp}(\tau)$ account for the contributions of $B_{\text {nuc }}^{z}$ and $B_{\text {nuc }}^{\perp}$ to the electron-spin precession. In the experimental range of the magnetic fields, the time dependence of $B_{\text {nuc }}^{z}$ is mainly caused by spectral diffusion due to the dipole-dipole interaction between nuclear spins. The weak effect 


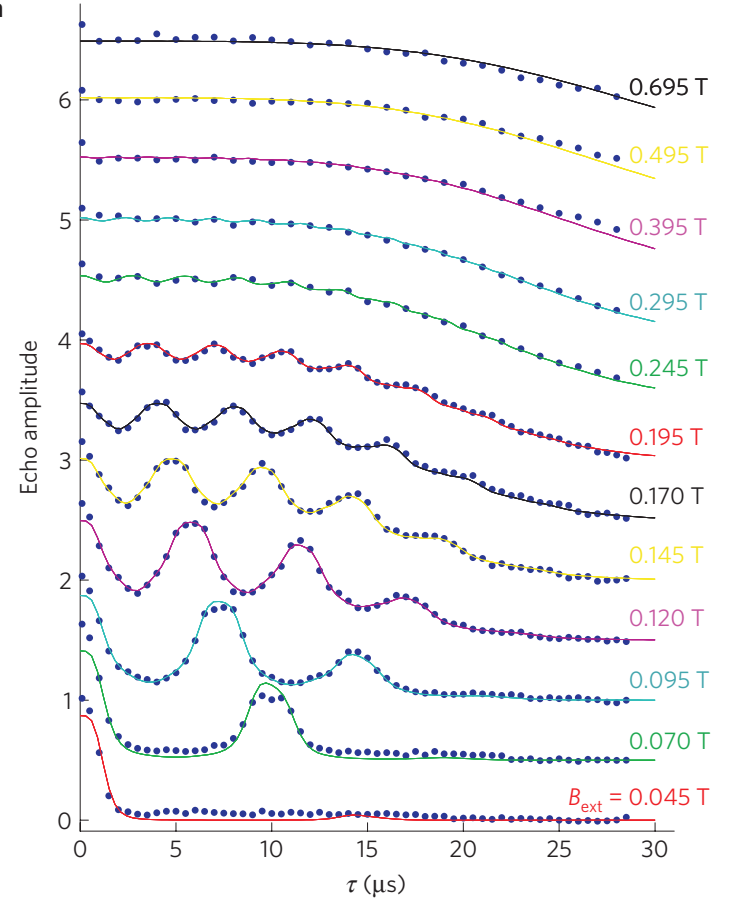

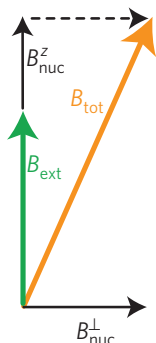

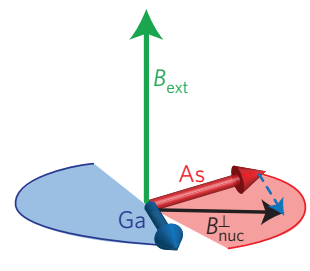

d

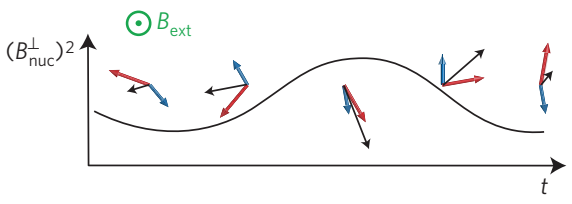

Figure 2 | Echo amplitude. a, Echo signal as a function of the total evolution time, $\tau$, for different values of magnetic field. The fits to the data are obtained by extending the model of ref. 3 to include a spread $\delta B_{\text {loc }}$ of the nuclear Larmor frequencies and multiplying by exp $\left(\left(-\tau / T_{S D}\right)^{4}\right)$. Curves are offset for

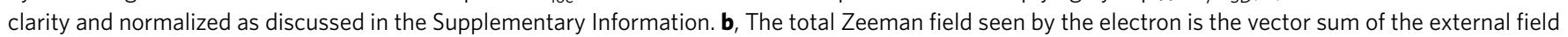
and the Overhauser fields parallel and perpendicular to it. c, The three nuclear species (only two shown for clarity) contributing to the Overhauser field precession at different Larmor frequencies in the external field. d, As a result of the relative precession, the total transverse nuclear field oscillates at the Larmor frequency difference(s).

of this generally slow process is predicted to lead to a decay of the form $A_{z}(\tau)=\exp \left(-\left(\tau / T_{\mathrm{SD}}\right)^{4}\right)$ (refs 13,15). As we will now discuss, $R_{\perp}(\tau)$ (see Supplementary Equation S1) has a more interesting non-monotonic structure that arises from the relative precession of nuclear spins in the external field with different Larmor frequencies.

The transverse hyperfine field, $\mathbf{B}_{\text {nuc }}^{\perp}$, is a vector sum of contributions from the three nuclear species ${ }^{69} \mathrm{Ga},{ }^{71} \mathrm{Ga}$ and ${ }^{75} \mathrm{As}$. As a result of the different precession rates of these species (Fig. 2c), $B_{\text {nuc }}^{\perp}(t)^{2}$ contains harmonics at the three relative Larmor frequencies (Fig. 2d) in addition to a constant term. The phase due to the constant term is eliminated by the echo pulse. For a general free-precession period, the time dependence leads to a suppression of the echo signal. However, if $\tau / 2$ is a multiple of all three relative Larmor periods, the oscillatory components contribute no net phase to the electron-spin evolution. As a result, the echo amplitude revives whenever the three commensurability conditions are approximately fulfilled, which is possible because of a fortuitous spacing of the Larmor frequencies. Averaging the singlet return probability over initial Overhauser field configurations leads to the collapse-and-revival behaviour predicted in refs 3,4 .

For $B_{\text {ext }} \lesssim 400 \mathrm{mT}$, the envelope of the echo revivals decays more quickly than at higher fields (see Fig. 2a). This field dependence can be accounted for by including a spread of the Larmor precession frequencies for each nuclear species. Such a variation is also manifest in the width of NMR lines and naturally arises from dipolar and other interactions between nuclei ${ }^{25}$. We model it as a shift of the magnetic field acting on each individual nuclear spin by an amount $B_{\text {loc }}$, where $B_{\text {loc }}$ is a Gaussian random variable with standard deviation $\delta B_{\text {loc }}$. This spread of precession frequencies leads to an aperiodic time dependence of $\left(B_{\text {nuc }}^{\perp}\right)^{2}$ that cannot be removed by the electron spin echo.

Using the above model (see also Supplementary Equation S1), we have fitted all of the data in Fig. $2 a$ with a single set of field-independent parameters that were chosen to obtain a good match with all data sets: the number of nuclei in each of the two dots, $N$, the spectral diffusion time constant, $T_{\mathrm{SD}}$, and $\delta B_{\mathrm{loc}}$. In addition, the scale factor for each data set was allowed to vary to account for the imperfect normalization of the data. The value of $N$ determines the depths of the dips between revivals. The best fit yields $N=4.4 \times 10^{6}$, which is in good agreement with an independent determination from a measurement of $T_{2}{ }^{*}=\sqrt{N} \hbar / g^{*} \mu_{\mathrm{B}} \cdot 4.0 \mathrm{~T}$ giving $N=4.9 \times 10^{6}$ (see ref. 26 and Supplementary Information). From the fit we also obtain $T_{\mathrm{SD}} \approx 37 \mu$ s and $\delta B_{\mathrm{loc}}=0.3 \mathrm{mT}$. The measured NMR linewidth in pure GaAs is about $0.1 \mathrm{mT}$ (ref. 25). A possible origin for the larger field inhomogeneity found here is the quadrupole splitting arising from the presence of the two localized electrons ${ }^{27}$. The inhomogeneity of the Knight shift is expected to have a similar but quantitatively negligible effect for our parameters. The value of $T_{\mathrm{SD}}$ is consistent with theoretical estimates ${ }^{15}$ (see Supplementary Information). Interestingly, the spread of nuclear Larmor frequencies, captured by $\delta B_{\text {loc }}$, contributes significantly to the echo decay even at the highest fields investigated. We have also verified that the Hahn-echo lifetime is not significantly affected by dynamic nuclear polarization, which can be used to increase $T_{2}{ }^{*}$ (see ref. 28 and Supplementary Information).

To measure the long Hahn-echo decay times of up to $30 \mu$ s, it was necessary to systematically optimize the pulses (see Supplementary Information). Small differences in the gate voltages before and after the $\pi$-pulse shift the electronic wavefunction relative to the inhomogeneously polarized nuclei. Such shifts cause the electrons to sample different Overhauser fields at different times, and thus lead to an imperfect echo. We have minimized this effect by compensating for a systematic drift of $\varepsilon$ over the course of each pulse sequence (see Supplementary Information).

Substantially longer coherence times are expected for more elaborate decoupling sequences ${ }^{17}$. We implemented the CPMG 

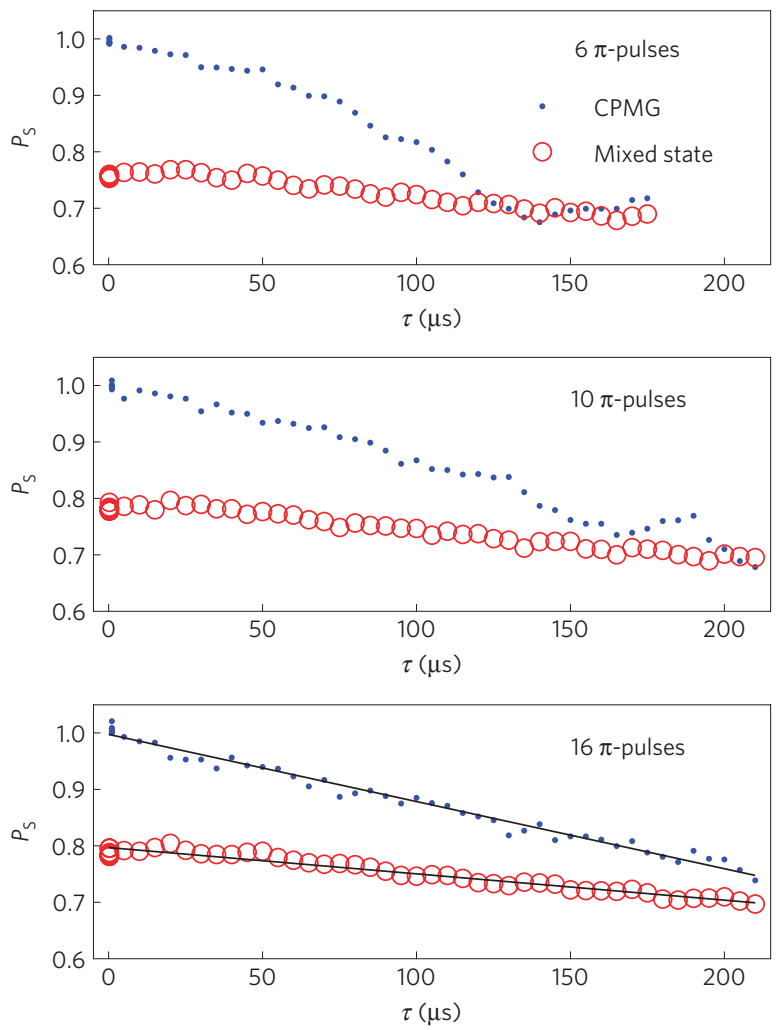

Figure 3 | CPMG decoupling experiments with 6,10 and $16 \pi$-pulses at $B_{\text {ext }}=0.4 \mathrm{~T}$. The blue dots show the readout signal of the CPMG pulses; the red circles represent reference measurements with the same evolution time without any $\pi$-pulses (equivalent to $T_{2}{ }^{*}$ measurements), which produce a completely dephased state. $P_{S}$ is the sensor signal normalized by the d.c. contrast associated with the transfer of an electron from one dot to the other, so that a singlet corresponds to $P_{S}=1$ (see Supplementary Information). Inelastic decay during the readout phase and possibly other visibility loss mechanisms increase $P_{S}$ compared with the actual singlet probability $p(S)$, so that the value for the mixed state exceeds the ideal value of 0.5 . The linear trends in the reference and the initial decay of the CPMG signal possibly reflect leakage out of the logical subspace. The linear fits to the 16-pulse data (black lines) intersect at $\tau=276 \mu$ s, which can be taken as a rough estimate or lower bound of the coherence time.

sequence $^{18}$, which consists of an $n$-fold repetition of the Hahn echo, thus requiring $n \pi$-pulses, as shown in Fig. 1d. Figure 3 shows data for $n=6,10$ and 16 . For $n=16$, the echo signal clearly persists for more than $200 \mu$ s. The field dependence for $n=4$ is reported in the Supplementary Information. To verify the interpretation of the data, we have measured the dependence of the echo on small changes in the final free-precession time and the duration of the exchange pulses for $n=10, \tau=5$ and $120 \mu$ s (Supplementary Information). As a result of the large number of potential tuning parameters, we have not optimized these CPMG pulses. We expect that with improved pulses the same extension of the coherence time could be achieved with fewer pulses. The initial linear decay of the signal in Fig. 3 is not well understood. The similar variation of the reference signal corresponding to a completely mixed state is suggestive of a single-electron $T_{1}$ process causing leakage into the $T^{+}$and $T^{-}$states (see Supplementary Information). The decay time constant sets a lower bound for the largest achievable coherence time.

Our measurements demonstrate coherence times of GaAs spin qubits of at least $200 \mu \mathrm{s}$, two orders of magnitude larger than previously shown. The duration of each of the $\pi$-pulses could easily be reduced below the $6 \mathrm{~ns}$ used here. One may hope to achieve millisecond-scale coherence times with improved decoupling sequences ${ }^{17}$ without adding complexity. Thus, $10^{5}-10^{6}$ operations could be carried out on one qubit while maintaining the state of another. However, more effort is required to realize pulses that use decoupling to improve the fidelity of short, nontrivial operations $s^{29,30}$, which seems feasible at least to lowest order. The excellent agreement with the model for the field and time dependence of the Hahn-echo revivals shows that many aspects of the dephasing of electron spins due to the nuclear hyperfine interaction are now well understood. The insight gained may also help pave the way towards probing macroscopic quantum effects in a mesoscopic ensemble of a few million nuclear spins.

Received 10 June 2010; accepted 20 October 2010; published online 12 December 2010

\section{References}

1. Petta, J. R. et al. Coherent manipulation of coupled electron spins in semiconductor quantum dots. Science 309, 2180-2184 (2005).

2. Koppens, F. H. L., Nowack, K. C. \& Vandersypen, L. M. K. Spin echo of a single spin in a quantum dot. Phys. Rev. Lett. 100, 236802 (2008).

3. Cywinski, L., Witzel, W. M. \& Das Sarma, S. Pure quantum dephasing of a solid-state electron spin qubit in a large nuclear spin bath coupled by long-range hyperfine mediated interactions. Phys. Rev. B 79, 245314 (2009).

4. Cywinski, L., Witzel, W. M. \& Das Sarma, S. Electron spin dephasing due to hyperfine interactions with a nuclear spin bath. Phys. Rev. Lett. 102, 057601 (2009).

5. Greilich, A. et al. Mode locking of electrons spin coherence in singly charged quantum dots. Science 313, 341-345 (2006).

6. Press, D., Ladd, T., Zhang, B. \& Yamamoto, Y. Complete quantum control of a single quantum dot spin using ultrafast optical pulses. Nature 456, 218-221 (2008).

7. Koppens, F. H. L. et al. Control and detection of singlet-triplet mixing in a random nuclear field. Science 309, 1346-1350 (2005).

8. Foletti, S., Bluhm, H., Mahalu, D., Umansky, V. \& Yacoby, A. Universa quantum control in two-electron spin quantum bits using dynamic nuclear polarization. Nature Phys. 5, 903-908 (2009).

9. Nowack, K. C., Koppens, F. H. L., Nazarov, Y. V. \& Vandersypen, L. M. K. Coherent control of a single electron spin with electric fields. Science 318, 1430-1433 (2007).

10. Barthel, C., Reilly, D. J., Marcus, C. M., Hanson, M. P. \& Gossard, A. C. Rapid single-shot measurement of a singlet-triplet qubit. Phys. Rev. Lett. 103, 160503 (2009).

11. Coish, W. A., Fischer, J. \& Loss, D. Exponential decay in a spin bath. Phys. Rev. B 77, 125329 (2008).

12. Chen, G., Bergman, D. L. \& Balents, L. Semiclassical dynamics and long-time asymptotics of the central-spin problem in a quantum dot. Phys. Rev. B 76, 045312 (2007).

13. Yao, W., Liu, R. B. \& Sham, L. J. Theory of electron spin decoherence by interacting nuclear spins in a quantum dot. Phys. Rev. B 74, 195301 (2006).

14. Childress, L. et al. Coherent dynamics of coupled electron and nuclear spin qubits in diamond. Science 314, 281-285 (2006).

15. Witzel, W. M. \& Das Sarma, S. Quantum theory for electron spin decoherence induced by nuclear spin dynamics in semiconductor quantum computer architecture. Phys. Rev. B 74, 035322 (2006).

16. Witzel, W. M. \& Das Sarma, S. Multiple-pulse coherence enhancement of solid state spin qubits. Phys. Rev. Lett. 98, 077601 (2007).

17. Lee, B., Witzel, W. M. \& Das Sarma, S. Universal pulse sequence to minimize spin dephasing in the central spin decoherence problem. Phys. Rev. Lett. 100, 160505 (2008).

18. Meiboom, S. \& Gill, D. Modified spin-echo method for measuring nuclear relaxation times. Rev. Sci. Instrum. 29, 688-691 (1958).

19. Khodjasteh, K. \& Lidar, D. A. Performance of deterministic dynamical decoupling schemes: Concatenated and periodic pulse sequences. Phys. Rev. A 75, 062310 (2007).

20. Uhrig, G. S. Keeping a quantum bit alive by optimizing $\pi$-pulse sequences. Phys. Rev. Lett. 98, 100504 (2007).

21. Biercuk, M. J. et al. Optimized dynamical decoupling in a model quantum memory. Nature 458, 996-1000 (2009).

22. Du, J. et al. Preserving electron spin coherence in solids by optimal dynamical decoupling. Nature 461, 1265-1268 (2009).

23. Levy, J. Universal quantum computation with spin-1/2 pairs and Heisenberg exchange. Phys. Rev. Lett. 89, 147902 (2002).

24. Field, M. et al. Measurements of Coulomb blockade with a noninvasive voltage probe. Phys. Rev. Lett. 70, 1311-1314 (1993).

25. Sundfors, R. K. Exchange and quadrupole broadening of nuclear acoustic resonance line shapes in the III-V semiconductors. Phys. Rev. 185, 458-472 (1969). 
26. Taylor, J. M. et al. Relaxation, dephasing, and quantum control of electron spins in double quantum dots. Phys. Rev. B 76, 035315 (2007).

27. Hester, R. K., Sher, A., Soest, J. F. \& Weisz, G. Nuclear-magnetic-resonance detection of charge defects in gallium arsenide. Phys. Rev. B 10, 4262-4273 (1974).

28. Bluhm, H., Foletti, S., Mahalu, D., Umansky, V. \& Yacoby, A. Enhancing the coherence of a spin qubit by operating it as a feedback loop that controls its nuclear spin bath. Phys. Rev. Lett. 105, 216803 (2010).

29. Viola, L., Lloyd, S. \& Knill, E. Universal control of decoupled quantum systems. Phys. Rev. Lett. 83, 4888-4891 (1999).

30. Khodjasteh, K. \& Viola, L. Dynamically error-corrected gates for universal quantum computation. Phys. Rev. Lett. 102, 080501 (2009).

\section{Acknowledgements}

We thank D. J. Reilly for advice on implementing the radiofrequency readout system and J. R. Maze for discussions. We acknowledge financial support from ARO/IARPA, the Department of Defense and the National Science Foundation under award number 0653336. I.N. and M.R. were supported by NSF grant DMR-0906475. This work was carried out in part at the Center for Nanoscale Systems (CNS), a member of the National Nanotechnology Infrastructure Network (NNIN), which is supported by the National Science Foundation under NSF award no. ECS-0335765.

\section{Author contributions}

Electron-beam lithography and molecular-beam-epitaxy growth were carried out by D.M. and V.U., respectively. H.B., S.F. and A.Y. fabricated the sample, planned and executed the experiment and analysed the data. I.N., M.R., H.B. and A.Y. developed the theoretical model. H.B., S.F., I.N., M.R. and A.Y. wrote the paper.

\section{Additional information}

The authors declare no competing financial interests. Supplementary information accompanies this paper on www.nature.com/naturephysics. Reprints and permissions information is available online at http://ngg.nature.com/reprintsandpermissions. Correspondence and requests for materials should be addressed to A.Y. 